\title{
The Gut Microbiome in Polycystic Ovary Syndrome and its Association with Metabolic
}

\section{Traits}

Kreete Lüll ${ }^{1,2}$, Riikka K. Arffman ${ }^{3}$, Alberto Sola-Leyva ${ }^{4,5}$, Nerea M. Molina ${ }^{4,5}$, Oliver Aasmets $^{1,2}$, Karl-Heinz Herzig ${ }^{6,7}$, Julio Plaza-Díaz ${ }^{8,9}$, Stephen Franks ${ }^{10}$, Laure MorinPapunen $^{3}$, Juha S. Tapanainen ${ }^{3,11}$, Andres Salumets ${ }^{12,13}$, Signe Altmäe $e^{4,5,12}$, Terhi T. Piltonen $^{3,14}$ and Elin Org ${ }^{*}$

${ }^{1}$ Institute of Genomics, Estonian Genome Centre, University of Tartu, Tartu, Estonia;

${ }^{2}$ Department of Biotechnology, Institute of Molecular and Cell Biology, University of Tartu, Tartu, Estonia;

${ }^{3}$ Department of Obstetrics and Gynecology, PEDEGO Research Unit, Medical Research Centre, Oulu University Hospital, University of Oulu, Oulu, Finland;

${ }^{4}$ Department of Biochemistry and Molecular Biology, Faculty of Sciences, University of Granada, Granada, Spain;

${ }^{5}$ Instituto de Investigación Biosanitaria ibs GRANADA, Granada, Spain;

${ }^{6}$ Research Unit of Biomedicine, Medical Research Center, University of Oulu; Oulu University Hospital, Oulu, Finland;

${ }^{7}$ Department of Paediatric Gastroenterology and Metabolic Diseases, Poznań University of Medical Sciences, Poznań, Poland;

${ }^{8}$ Department of Biochemistry and Molecular Biology II, Faculty of Pharmacy, University of Granada, Granada, Spain;

${ }^{9}$ Children's Hospital of Eastern Ontario Research Institute, Ottawa ON K1H 8L1, Canada; 
${ }^{10}$ Faculty of Medicine, Department of Metabolism, Digestion and Reproduction, Imperial College London, London, United Kingdom;

${ }^{11}$ Department of Obstetrics and Gynecology, University of Helsinki and Helsinki University Hospital, Helsinki, Finland;

${ }^{12}$ Department of Obstetrics and Gynaecology, Institute of Clinical Medicine, University of Tartu, Tartu, Estonia;

${ }^{13}$ Competence Centre on Health Technologies, Tartu, Estonia;

${ }^{14}$ Department of Obstetrics and Gynecology, Reproductive Endocrinology and IVF Unit, Oulu, University Hospital, University of Oulu, Oulu, Finland.

*Corresponding author:

Elin Org, $\mathrm{PhD}$

Institute of Genomics, Estonian Genome Centre

University of Tartu

Tartu 51010, Estonia

phone: (+372) 737 4034; fax: (+372) 737 4060; email: elin.org@ut.ee

Funding. This work was funded by Estonian Research Council grants PUT 1371 (to EO), IUT34-16 (to AS), Enterprise Estonia grant No. EU48695 (to AS), and EMBO Installation grant 3573 (to EO and supported KL, OA). EO was supported by European Regional Development Fund Project No. 15-0012 GENTRANSMED, Estonian Center of Genomics/Roadmap II project No. 16-0125 and Estonian Research Council grant PRG 687. KL was supported by The European Regional Development Fund. SA was supported by the Spanish Ministry of Economy, Industry and Competitiveness (MINECO) and European Regional Development Fund (FEDER): grants RYC-2016-21199 and ENDORE (SAF201787526-R); and by FEDER/Junta de Andalucía-Consejería de Economía y Conocimiento: 
MENDO (B-CTS-500-UGR18). ASL and NMM are funded by the Spanish Ministry of Science, Innovation and Universities: PRE2018-0854409 (ASL) and FPU19/01638 (NMM). TTP and RKA are funded by Finnish Academy and Sigrid Juselius Foundation. NFBC1966 received financial support from University of Oulu Grant No. 65354, Oulu University Hospital Grant No. 2/97, 8/97, Ministry of Health and Social Affairs Grant No. 23/251/97, 160/97, 190/97, National Institute for Health and Welfare, Helsinki Grant No. 54121, Regional Institute of Occupational Health, Oulu, Finland Grant No. 50621, 54231. NFBC1966 received financial support from University of Oulu Grant No. 24000692, Oulu University Hospital Grant No. 24301140, ERDF European Regional Development Fund Grant No. 539/2010 A31592.

Disclosure Summary: The authors declare nothing to disclose. 


\section{ABSTRACT}

Context: Despite gut microbiome being widely studied in metabolic diseases, its role in polycystic ovary syndrome (PCOS) has been scarcely investigated.

Objective: Compare the gut microbiome in late fertile age women with and without PCOS and investigate whether changes in the gut microbiome correlate with PCOS-related metabolic parameters.

Design: Prospective, case-control study using the Northern Finland Birth Cohort 1966.

Setting: General community.

Participants: 102 PCOS women and 201 age- and body mass index (BMI)-matched non-PCOS control women. Clinical and biochemical characteristics of the participants were assessed at ages 31 and 46 and analyzed in the context of gut microbiome data at the age of 46.

\section{Intervention(s): None}

Main outcome measure(s): Bacterial diversity, relative abundance, and correlations with PCOS-related metabolic measures.

Results: Bacterial diversity indices did not differ significantly between PCOS and controls (Shannon diversity $\mathrm{p}=0.979$, unweighted UniFrac $\mathrm{p}=0.175$ ). Four genera whose balance helps to differentiate between PCOS and non-PCOS were identified. In the whole cohort, the abundance of two genera from Clostridiales, Ruminococcaceae UCG-002 and Clostridiales Family XIII AD3011 group, were correlated with several PCOS-related markers. Prediabetic PCOS women had significantly lower alpha diversity (Shannon diversity $\mathrm{p}=$ 
$0.018)$ and markedly increased abundance of genus Dorea (FDR $=0.03)$ compared to women with normal glucose tolerance.

Conclusion: PCOS and non-PCOS women at late fertile age with similar BMI do not significantly differ in their gut microbial profiles. However, there are significant microbial changes in PCOS individuals depending on their metabolic health.

Key Words: PCOS, gut microbiome, metabolic traits, type 2 diabetes 


\section{INTRODUCTION}

Polycystic ovary syndrome (PCOS) is one of the most prevalent endocrine and metabolic disorders in women, affecting $8-18 \%$ of women in reproductive age, depending on the studied population and applied diagnostic criteria (1-3). PCOS is characterized by persistent menstrual irregularities, clinical or biochemical hyperandrogenism and polycystic ovarian morphology. It is a complex disorder associated with a variety of metabolic derangements, including obesity, insulin resistance (IR) and type 2 diabetes (T2D). However, regardless of obesity, IR is present in up to $50 \%$ of women with PCOS $(4,5)$. The etiology of PCOS remains unknown but is believed to be multifactorial where genetics, intrauterine environment, lifestyle factors and possibly alterations in the gut microbiome could have a role (6).

The human gut microbiota refers to all of the microorganisms inhabiting the gastrointestinal tract (7), where the majority belong to four bacterial phyla - Bacteroidetes, Firmicutes, Proteobacteria and Actinobacteria (8). Improved metabolic health has been related to increased microbial diversity and diverse microbiome gene content (9), while changes in the gut microbiome composition have been associated with a vast number of diseases and disorders beyond the gastrointestinal health, including T2D (10-13), obesity (14-17), IR $(18,19)$ and depression (20-22), among others.

Recently, the possible link between PCOS and gut microbiome has drawn increased attention. Previous studies investigating the relationship between gut microbiome and PCOS in women of different ethnicities have reported reduced bacterial diversity and altered overall composition as well as changes in the relative abundance of specific bacteria, mostly belonging to Bacteroidetes and Firmicutes phyla (23-27). Nevertheless, despite the reported links between the gut microbiota and clinical parameters in PCOS, the results are inconsistent 
and the community structure and function of the gut microbiome in women with PCOS remains unclear. Moreover, lack of body mass index (BMI) adjustment or BMI-matched controls, small sample size, ethnic differences, and variability in the methods used when conducting the microbiome analysis can lead to difficulties in reproducing the results. Hence, population-based cohorts, in which individuals have been evaluated during the follow-up visits, provide an important resource to define homogeneous phenotypes and detect changes in multiple pathophysiological pathways. Previous studies have included PCOS women of reproductive age, whereas the effects of the microbiome on the health of women during the late reproductive and pre- or early menopausal years have remained unstudied.

The aim of our study was to test whether gut microbiome is associated with PCOS in late fertile age women including age- and BMI-matched controls. We also aimed to assess different metabolic and hormonal markers related to PCOS and their associations with the gut microbiome.

\section{MATERIAL AND METHODS}

\section{Study population}

The study population consisted of a subset of females in the longitudinal Northern Finland Birth Cohort 1966 (NFBC1966) which includes all expected births in 1966 in the two northernmost provinces of Finland $(28,29)$. The NFBC1966 is a unique population-based cohort, which was established as a longitudinal research program to promote health and wellbeing of the population. Data collection procedures and the identification of PCOS cases in the NFBC1966 have been described earlier (30-32). A flow-chart of the sample selection process is represented in Supplementary Figure 1 (33). In brief, at age 31 a questionnaire was sent to all women with known addresses in Finland and a majority of them also participated in a clinical examination with anthropometric measurements and blood samples drawn and 
analyzed. At age 46 the women were invited for a follow-up and to fill in a questionnaire. During the latter follow-up, clinical examinations with blood and fecal sample collection, oral glucose tolerance test (OGTT) and anthropometric measurements were performed. The participants received no specific instructions or recommendations regarding their diet prior to fecal sample collection. Women with PCOS in the cohort were identified based on the questionnaires at ages 31 and 46. Briefly, at age of 31, women were asked whether they had oligo-amenorrhea (OA) or hirsutism. If they answered "yes" to both, they were considered as PCOS cases. At 46, instead of OA and hirsutism questions, the women were asked if they had been diagnosed with polycystic ovaries (PCO) and/or PCOS and those answering "yes" were considered PCOS cases. The total PCOS population consisted of women who reported both hirsutism and OA at age 31 and/or PCO/PCOS at age 46. The validity of the PCOS diagnosis using the method described above has been verified in previous publications $(31,32,34)$. The non-PCOS control population consisted of women with no PCOS symptoms at 31 nor PCO/PCOS by 46 years of age. Women who were on hormonal contraceptives or were pregnant at 31 years were excluded. The Finnish register for drug reimbursements was used to identify women who had been prescribed antibiotics, antimycotics, letrozole or tamoxifen within the 3 months preceding sample collection, who were then excluded. Also, women not permitting the use of their data were excluded. Two BMI-matched controls were chosen for each woman with PCOS. Women with PCOS from whom fecal samples were available were identified from the dataset. The total study population for the current study was 304 women with 102 women with PCOS and 202 non-PCOS control women. The study has been approved by the ethical committee of Northern Ostrobothnia hospital district. All participants of the NFBC1966 have given informed consent for the data and samples to be used for scientific purposes. 


\section{Sampling and laboratory measurements}

\section{Hormonal measurements}

Testosterone (T) was assayed from serum using Agilent triple quadrupole 6410 liquid chromatography-mass spectrometry equipment (Agilent Technologies, Wilmington, DE, USA). Elevated T level at ages 31 and 46 was defined according to the normal upper limit for $\mathrm{T}$ at these respective ages based on the $97.5 \%$ percentile calculated in this population $(2.3$ $\mathrm{nmol} / \mathrm{L}$ at age 31 and $1.7 \mathrm{nmol} / \mathrm{L}$ at age 46). Consequently, at age 31 , women with $\mathrm{T}>2.3$ $\mathrm{nmol} / \mathrm{L}$ were considered to have elevated $\mathrm{T}$ levels and those with $\mathrm{T} \leq 2.3 \mathrm{nmol} / \mathrm{L}$ as women with normal $\mathrm{T}$ levels. An elevated $\mathrm{T}$ level at age 46 was defined as serum level of $\mathrm{T}>1.7$ nmol/L and normal $\mathrm{T}$ level as serum $\mathrm{T} \leq 1.7 \mathrm{nmol} / \mathrm{L}$. Sex hormone binging globulin $(\mathrm{SHBG})$ was assayed by chemiluminometric immunoassay (Immulite 2000, Siemens Healthcare, Llanberis, UK). The free androgen index (FAI) was calculated using the following equation: $\mathrm{T}(\mathrm{nmol} / \mathrm{L}) / \mathrm{SHBG}(\mathrm{nmol} / \mathrm{L}) \times 100$. For more detailed information please see Ollila et al . $2016(32)$.

\section{Glucose metabolism assessment}

Plasma glucose was analyzed by an enzymatic dehydrogenase method (Advia 1800, Siemens Healthcare Diagnostic Inc., Tarrytown, NY, USA). Serum insulin was analyzed by a chemiluminometric immunoassay (Advia Centaur XP, Siemens Healthcare Diagnostics, Tarrytown, NY, USA). A 75 g glucose OGTT was performed with blood glucose and insulin measurements were taken before and after $30 \mathrm{~min}, 60 \mathrm{~min}$ and $2 \mathrm{~h}$ of glucose intake. Area under the curve (AUC) for glucose and AUC for insulin were calculated using the equation $[($ fasting $X+(2 \times(30 \min X)+(3 \times 60 \min X)+(2 \times 120 \min X)] \times 15$, where $X$ states glucose $(\mathrm{mmol} / \mathrm{L})$ or insulin $(\mu \mathrm{U} / \mathrm{mL})$. The secretion index was calculated using the equation (fasting insulin $(\mu \mathrm{U} / \mathrm{mL})+30 \mathrm{~min}$ insulin $(\mu \mathrm{U} / \mathrm{mL})) \times 6.945 /($ fasting glucose $(\mathrm{mmol} / \mathrm{L})+$ 
$30 \mathrm{~min}$ glucose $(\mathrm{mmol} / \mathrm{L}))$. Matsuda index was calculated using the equation $10000 / \mathrm{sqrt}$ $(($ fasting insulin $(\mu \mathrm{U} / \mathrm{mL}) \times$ fasting glucose $(\mathrm{mmol} / \mathrm{L}) \times 18) \times($ AUC glucose $\times 18 / 120 \times$ AUC insulin / 120)) $(32,35)$. The disposition index was calculated by multiplying the secretion index by Matsuda index.

Individuals were grouped according to WHO classification of diabetes based on their OGTT results (36). A person was categorized as preT2D if they had impaired fasting glucose (IFG: fasting plasma glucose from 6.1 to $6.9 \mathrm{mmol} / \mathrm{L}$ ), impaired glucose tolerance (IGT: $2 \mathrm{~h}$ glucose from 7.8 to $11.0 \mathrm{mmol} / \mathrm{L}$ ), or both. A person was considered diabetic if they had fasting plasma glucose over $7.0 \mathrm{mmol} / \mathrm{L}$ or $2 \mathrm{~h}$ plasma glucose over $11.2 \mathrm{mmol} / \mathrm{L}$. Based on BMI scores, individuals were assigned into BMI groups: underweight (BMI below 18.4), normal weight (BMI 18.5-24.9), overweight (BMI 25-29.9), and obese (BMI over 30) [Supplementary Table $1(33)]$.

\section{Gut microbiome analysis}

Fecal samples were collected at home by the study participants at age 46 . It was recommended that the fecal sample should be delivered in a cooler on the day of collection. If that was not possible, the sample was stored for one or two days in a freezer at $-20^{\circ} \mathrm{C}$ until delivery. After delivery, the fecal samples were initially stored at $-20^{\circ} \mathrm{C}$ and then moved to $70^{\circ} \mathrm{C}$ for long-term storage.

For bacterial DNA isolation, first the samples were homogenized in a Stomacher-400 blender. QIAamp Stool Mini Kit (Qiagen, Venlo, The Netherlands) was used for DNA extraction. The standard protocol was followed, with the exception that the samples were mixed with the lysis buffer and incubated at $95^{\circ} \mathrm{C}$ instead of $70^{\circ} \mathrm{C}$ in order to ensure the lysis of both Gram-negative and Gram-positive bacteria. The extracted DNA was quantified with NanoDrop ND-1000 spectrophotometer (Thermo Fisher Scientific, DE, USA). DNA yield 
was determined by measuring absorbance ratios spectrophotometrically, and included A260/280 nm for protein and A260/230 nm for salt and phenol contamination.

Microbiome was profiled by sequencing the V3-V4 regions of the 16S rRNA gene on an Illumina MiSeq instrument with forward 5'-CCTACGGGNGGCWGCA-3' and reverse 5'-GACTACHVGGGTATCTAATCC-3' primers. All PCR reactions were performed in 25 $\mu \mathrm{l}$ volume containing $12.5 \mu \mathrm{l}$ 2X KAPA HiFi Hotstart ready mix (KAPA Biosystems, Woburn, MA, USA), $5 \mu \mathrm{L}$ of each primer $(1 \mu \mathrm{M})$, and $2.5 \mu \mathrm{L}$ of extracted DNA (10 ng) under the following cycling conditions: initial denaturation at $95^{\circ} \mathrm{C}$ for $3 \mathrm{~min}$, followed by 35 cycles of denaturation at $95^{\circ} \mathrm{C}$ for $30 \mathrm{~s}$, annealing at $55^{\circ} \mathrm{C}$ for $30 \mathrm{~s}$, and elongation at $72^{\circ} \mathrm{C}$ for $30 \mathrm{~s}$, with a final extension at $72^{\circ} \mathrm{C}$ for $5 \mathrm{~min}$. PCR clean-up was done with AMPure XP beads (Beckman Coulter, Indianapolis, IN, USA). Next, a PCR to index the amplicons was performed using the Nextera XT Index Kit (Illumina, San Diego, CA, USA) with conditions: $95^{\circ} \mathrm{C}$ for $3 \mathrm{~min} ; 8 \mathrm{cycles}$ of $95^{\circ} \mathrm{C}$ for $30 \mathrm{~s}, 55^{\circ} \mathrm{C}$ for $30 \mathrm{~s}, 72^{\circ} \mathrm{C}$ for $30 \mathrm{~s}$, with a final extension step of $5 \mathrm{~min}$ at $72^{\circ} \mathrm{C}$, and hold at $4^{\circ} \mathrm{C}$. Next, the pooled PCR products were purified using AMPure XP beads (Beckman Coulter) before quantification. The final library was paired-end sequenced ( 2 x 300 bp) using a MiSeq Reagent Kit v.3 on the Illumina MiSeq sequencing system (Illumina).

\section{S rRNA sequencing data analysis}

Raw sequences were demultiplexed with Illumina bcl2fastq2 Conversion Software v2.20 and raw data were imported into open-source software QIIME 22019.7 (37) using the q2-tools-import script with PairedEndFastqManifestPhred33 input format. In total, 16,063,617 (average 78,743 per person) reads were generated with the V3-V4 16S rRNA sequencing. Denoising was done with DADA2 (38), using a quality-aware model of Illumina amplicon errors to attain an abundance distribution of sequence variances, which have a 
difference of a single nucleotide. After retrieving quality scores, q2-dada2-denoise script was used to truncate the forward reads at position 288 and trim at position 16. Reverse reads were truncated at position 241, trimming was not applied on the reverse read. Chimera removal was done using the "consensus" filter via q2-dada2-denoise in which chimeras are detected in samples individually and sequences found chimeric in a sufficient fraction of samples are removed. During this step forward and reverse reads are also merged. All amplicon sequence variants (ASVs) were aligned with MAFFT (39) via q2- alignment and phylogeny was constructed with FASTTREE2 (via q2- phylogeny) (40). Taxonomy was assigned to ASVs using the classify $\square$ sklearn naïve Bayes taxonomy classifier (via q2- feature- classifier) (41) against the SILVA 16S V3-V4 v132_99 (42) along with a similarity threshold of 99\%. Altogether, 72,738 ASVs were identified (average of 680 ASVs for women with PCOS, and average of 670 ASVs for controls). A total number of 399 genera, 127 families, 56 orders, 29 classes and 19 phyla were detected. Data filtering steps included pruning samples to exclude samples with less than 10,000 reads after which 303 samples were left (102 women with PCOS and 201 control women).

\section{Statistical analysis}

Statistical analyses and data visualization were performed using the statistical software R v.3.6.1 (under RStudio v.1.2.1335). All of the visualizations were made using the ggplot2 v.3.3.0 (43) and corrplot v.084 (44) packages. A p-value below 0.05 was considered to be statistically significant and multiple testing was taken into account using the Benjamini and Hochberg False Discovery Rate (FDR) method (45). For beta diversity and association analysis we filtered out taxa that were detected in less than $30 \%$ of the remaining samples. The data were aggregated to genus level for further analysis. Alpha (Shannon's index, observed, and inverse Simpson's index) and beta [Principal Coordinate Analysis (PCoA), 
based on the UniFrac distance metrics (unweighted and weighted)] diversities were calculated using the phyloseq v.1.28.0 package (46). Weighted UniFrac metric accounts for taxa abundances in calculating the distances, whereas unweighted UniFrac only takes into account whether the taxa are present/absent and does not put emphasis on microbial abundances. For diversity comparisons, alpha diversity estimators were calculated and evaluated using ANOVA, one-way ANOVA tests with Tukey multiple pairwise-comparisons as appropriate. ADONIS-2 function from the vegan package using 10,000 permutations for pvalue calculations was used when testing differences in beta diversity. In the clinical biomarker's data comparison, the values were expressed as mean \pm standard deviation. Wilcoxon sign rank test was used for testing the differences in the continuous variables between the study groups. Statistical analysis between groups of categorical variables were analyzed by Fisher's Exact test and the data were indicated as relative and absolute frequencies. The core microbiome was identified using the microbiome v.1.6.0 package (47) with a detection threshold of $0.01 \%$ and prevalence threshold of $95 \%$. Associations between alpha diversity and clinical biomarkers were tested using Spearman correlation and adjusting for FDR using the cor function from the WGCNA v.1.69.package (48). ANOVA-Like Differential Expression tool (ALDEx2 v.1.16.0) (49) was used to identify differentially abundant taxa. Selbal v.0.1.0 (50) was used to identify taxa whose balance, a log-contrast between two groups of taxa was predictive of PCOS status. Correlation analyses between taxa and biomarkers were performed using the function bicorAndPvalue from the WGCNA v.1.69 package (48). 


\section{RESULTS}

\section{Clinical characteristics of study participants}

A total of 303 women were included for analysis (102 PCOS and 201 age- and BMImatched healthy controls). Table 1 summarizes the clinical characteristics of the study participants. Testosterone at 31 years $(\mathrm{p}=0.01)$ and FAI at 46 years $(\mathrm{p}=0.01)$ were higher in women with PCOS when compared with controls. As expected, matching for BMI resulted in a lack of significance in the difference in SHBG between the study groups, although the SHBG still showed some tendency for being lower in PCOS. Compared with controls, women with PCOS reported having fewer menses per year at age 31 and they experienced more infertility problems (lifetime) by age 31 and 46 years.

\section{Landscape of microbiome composition and diversity}

We first characterized the phylogenetic variation across all the samples $(n=303)$ at different taxonomic levels. We detected in total 72,738 ASVs, which resulted in a total richness of 399 genus-level taxonomic groups. After filtering out genus present in less than $30 \%$ of the samples, the data consisted of 128 genera, 37 families, 18 orders, 14 classes and 8 phyla. We observed a typical Western diversity profile for gut microbiota, where Firmicutes (54.0\%) and Bacteroidetes (31.9\%) were the dominant phyla, followed by Proteobacteria (6.7\%), Actinobacteria (3.4\%), and Verrucomicrobia (2.4\%) (Fig 1 A). The core microbiome (the number of taxa present in over $95 \%$ of individuals across the whole cohort) represent eight different genera belonging to two most abundant phyla, Bacteroidetes and Firmicutes. The most abundant genus was Bacteroides (19.9\%), followed by Alistipes (7.5\%), Faecalibacterium (4.9\%), Roseburia (2.5\%), Blautia (2.5\%), Lachnoclostridium (1.5\%), Ruminococcaceae uncultured (1.2\%), and Oscillibacter (1.1\%) (Fig 1 B). 
Next, we assessed differences in the gut microbiome diversity (alpha and beta diversities) between controls and PCOS samples. In alpha diversity analyses, that assesses the richness and evenness of the bacterial community, PCOS subjects showed no differences from the controls in any of the assessed metrics $\left(\mathrm{P}_{\text {Shannon }}=0.979, \mathrm{P}_{\text {InverseSimpson }}=0.248\right.$, $\left.\mathrm{P}_{\text {Observed }}=0.870\right)[$ Fig 2A, Supplementary Fig 2 (33)]. Beta diversity, which represents how much the community changes between controls and PCOS samples, the PCoA based on both unweighted UniFrac (ADONIS $\mathrm{p}=0.175, \mathrm{r}^{2}=0.004$ ) and weighted UniFrac measures (ADONIS $\mathrm{p}=0.44, \mathrm{r}^{2}=0.003$ ), did not show any clustering with PCOS nor difference between the PCOS and non-PCOS groups (Fig 2B, C).

We then investigated whether bacterial richness (Shannon index, as alpha diversity) and beta diversity were associated with clinical, hormonal and metabolic parameters for all women. The Shannon index was negatively correlated with BMI $($ FDR $=0.006)$, fasting insulin $($ FDR $=0.01)$ and FAI $($ FDR $=0.02)$ and positively correlated with SHBG $($ FDR $=$ 0.0007), Matsuda Index $($ FDR $=0.0007)$ and Disposition Index $($ FDR $=0.007)$ [Supplementary Table 2 (33)]. Both unweighted and weighted UniFrac had statistically significant associations with BMI $\left(\mathrm{FDR}_{\text {weighted }}=6.4 \times 10^{-4}, \mathrm{FDR}_{\text {unweighted }}=5.3 \times 10^{-4}\right)$, fasting insulin $\left(\mathrm{FDR}_{\text {weighted }}=0.01, \mathrm{FDR}_{\text {unweighted }}=0.003\right)$, Matsuda Index $\left(\mathrm{FDR}_{\text {weighted }}=5.3 \times 10^{-4}\right.$, $\left.\mathrm{FDR}_{\text {unweighted }}=6.4 \times 10^{-4}\right)$, Disposition Index $\left(\mathrm{FDR}_{\text {weighted }}=5.3 \times 10^{-4}, \mathrm{FDR}_{\text {unweighted }}=0.01\right)$, and SHBG $\left(\mathrm{FDR}_{\text {weighted }}=0.007, \mathrm{FDR}_{\text {unweighted }}=0.0068\right)[$ Supplementary Table $2(33)]$.

\section{Differences in the gut microbiome between the healthy women and women with}

\section{PCOS}

The relative abundance of the 128 genera (present at least in $30 \%$ of the population) were compared between healthy control women and PCOS women [Supplementary Table 3 (33)]. First, we used the ALDEx2 package (v1.16.0), which performs differential abundance 
analysis for the comparison of two or more conditions, PCOS vs. non-PCOS in this case (49). We did not observe any genera that showed statistically significant differences between the PCOS and control women after correcting for multiple testing, the top ten genera identified with ALDEx2 are shown in Supplementary Table 4 [Supplementary Table 4 (33)].

With the Selbal analysis, we aimed to identify microbial signatures - groups of microbial taxa that are able to differentiate between PCOS and non-PCOS women. The identification of microbial signatures involves modelling the response variable (response being PCOS or control) and identifying the smallest number of taxa with the highest prediction accuracy (50). Therefore, these microbial signatures could be used to identify PCOS cases based on an individual's specific microbiome. Selbal analysis identified two groups of taxa consisting of Paraprevotella-Streptococcus and Eubacterium ventriosum group-Bifidobacterium, whose relative abundance or balance could differentiate between PCOS women and control women (Fig 2D). The discrimination value of the balance (AUC = 0.643) demonstrates a modest discrimination between the PCOS and healthy women. Interestingly, the Eubacterium ventriosum group, Paraprevotella, and Streptococcus were also among the top 10 genera that we identified in the ALDEx2 analysis [Supplementary Table 4 (33)]. However, Selbal cannot be compared with ALDEx2 in terms of power and FDR since the aim of Selbal is to obtain the best model to predict the response in contrast to identifying all taxa that are associated with the response, which is the aim of ALDEx2.

\section{Associations of taxa with hormonal and metabolic markers}

We correlated the top ten associated taxa [Supplementary Table 4 (33)] to the markers that could possibly have a role in the development of PCOS. The abundance of genus Ruminococcaceae UCG-002 was positively correlated with Matsuda Index ( $\mathrm{p}=0.009$; FDR $=0.140)$, Disposition Index $(p=0.001 ;$ FDR $=0.065)$ and SHBG $(p=0.001 ;$ FDR $=0.065)$. 
The abundance of Clostridiales Family XIII AD3011 group was positively correlated with Matsuda Index $(p=0.010 ;$ FDR $=0.140)$ and SHBG $(p=0.006 ;$ FDR $=0.140)$ and negatively correlated with glycated haemoglobin HbA1c $(\mathrm{p}=0.003$; FDR $=0.099), 2 \mathrm{~h}$ glucose level $(\mathrm{p}=0.006 ; \mathrm{FDR}=0.140)$ and BMI $(\mathrm{p}=0.010 ; \mathrm{FDR}=0.140)[$ Fig 3 , Supplementary Table 5 (33)].

\section{Relationships between PCOS and metabolic traits}

Previous studies have shown that obesity has a critical role in PCOS and individuals with PCOS have an elevated risk in developing T2D (51). Therefore, we next aimed to evaluate the relationships between the gut microbiome and their potential effect on BMI and T2D in PCOS individuals. In order to study the relationships of PCOS and T2D together with gut microbiome, we focused on the women with PCOS and categorized them as having a normal glucose tolerance (NGT) or preT2D based on the values of fasting and $2 \mathrm{~h}$ plasma glucose following the OGTT. The preT2D state includes individuals with IFG, IGT, or both (see "Methods" for details). In the PCOS group, there were 76 NGT individuals and 14 preT2D individuals. The four individuals who already had developed T2D based on their OGTT and eight individuals with no OGTT data were excluded from this analysis. We compared microbiota composition between PCOS women with NGT $(\mathrm{n}=76)$ and with preT2D $(\mathrm{n}=14)$. In diversity analyses, both alpha (Shannon index; $\mathrm{p}=0.018)$ and beta diversities (unweighted UniFrac; $p=0.003$ and weighted UniFrac; $p=0.012$ ) showed

statistically significant differences between the NGT and preT2D in the PCOS group [Fig 4 A, B; Supplementary Fig 3 (33)]. These results are in line with previous studies which also report decreased microbial diversity in preT2D and T2D compared with NGT (52). As weighted UniFrac, opposed to unweighted UniFrac, takes abundance into account, these results indicate that the association between preT2D and human gut microbiome could be 
induced by the presence/absence of more abundant taxa. In contrast, when analyzing control women, we did not observe differences in alpha or beta diversities between NGT and preT2D women (data not shown).

We also detected differentially abundant taxa between the NGT and preT2D in PCOS women, where the genus Dorea (Fig 4C) had elevated abundance in preT2D with the difference being statistically significant after FDR correction $(F D R=0.03)$. Genera Bacteroides $(\mathrm{FDR}=0.07)$, Ruminococcus torques group $(\mathrm{FDR}=0.07)$ and Lachnospiraceae UCG-004 $(\mathrm{FDR}=0.06)$ reported results of borderline significance [Supplementary Table 6, Supplementary Fig 4 (33)]; all three had elevated abundance in preT2D group.

To study the effect of BMI on gut microbiome, we divided the PCOS individuals into three subgroups based on their BMI values: normal weight $(n=37)$, overweight $(n=38)$, and obese $(n=27)$. There were no statistically significant differences in diversity analysis (both alpha and beta diversity) between any of the BMI sub-groups [Supplementary Fig 5 A-C (33)]. ALDEx2 analysis revealed no statistically significant taxa differences after correcting for multiple testing in between the three BMI groups [Supplementary Table 7 (33)].

\section{DISCUSSION}

In this study, we investigated the relationship of gut microbiome with PCOS status in women nearing the end of their fertile age, by assessing a total of 303 women from the population-based NFBC1966. We first characterized the gut microbiome diversity and composition of the whole study cohort. The gut microbiome profile for Finnish females in their late fertile years is representative of the average gut microbiome profile in a population consuming a so-called Western-diet with the most prevalent phyla being Firmicutes and Bacteroidetes, followed by Proteobacteria, Actinobacteria, and Verrucomicrobia (53). The core microbiome (i.e., the genera shared by $95 \%$ of samples) in our cohort consists of 8 
genera, of which 6 overlapped with the recent international MiBioGen consortium study findings where a total of 18,473 fecal samples across 25 populations were analysed (54). Only two additional taxa, Oscillibacter and Ruminococcaceae uncultured, were identified as core members in our cohort. Both gender effect and geographic differences might explain the observed differences in core gut microbiota.

Next, we assessed the diversity and taxonomic differences between the women with PCOS and non-PCOS controls. The fecal samples for gut microbiome analysis were only collected at the age of 46. Diversity analysis showed no significant difference in bacterial alpha and beta diversities between PCOS women and healthy control women. In a previous work, Qi et al. also reported no differences in alpha diversity when using whole-genome shotgun sequencing between the PCOS and controls, however, they did report significantly lower beta diversity in PCOS compared to the controls (55). Diversity differences between the PCOS and non-PCOS individuals are inconsistent throughout previous studies; while some were able to detect changes in alpha/beta diversities $(23,24,26,27,56)$, others found no significant differences between groups $(25,55,57,58)$. It is possible that PCOS itself does not alter the gut microbiome to such extent that it reflects changes in the whole microbiome community. Rather the changes in diversity may become significant when analyzing PCOSrelated metabolic traits. We did observe significant associations between diversity measures and PCOS-related hormonal and metabolic parameters such as BMI, SHBG levels, and insulin sensitivity as well as IR as measured by Matsuda and disposition indices in the entire cohort of women. 
Despite seeing no significantly different taxa between the PCOS and controls after multiple corrections, some of the top genera have previously been linked with PCOS-related traits. Eubacterium ventriosum group, that we detected in both single taxa and group-based modelling analyses, has previously shown to be enriched in obese individuals (59). Another genus that we detected both in Selbal as well as in ALDEx analysis was Streptococcus. Liu et al. reported in their study that bacteria belonging to Streptococcus belonged to co-abundance groups that were increased in PCOS idividuals and were positively correlated with BMI and testosterone (25). The genus Paraprevotella has been formerly detected in prenatal androgenized rat model where Paraprevotella was significantly enriched in androgenized rodents $(60,61)$. The fact that many PCOS cases also suffer from hyperandrogenism may explain the link between genus Paraprevotella and PCOS. However, in our results Paraprevotella was decreased in PCOS women. It is possible that this discrepancy is due to the fact that the women were already 46 years old and that their androgen levels did not differ anymore from the age- and BMI-matched controls at this time point. The genus Turicibacter itself has not been reported to be associated with PCOS previously, but in the present analysis it was one of the top genera detected in PCOS. Turicibacter belongs to the family Erysipelotrichaceae that has been reported to have elevated levels in PCOS both in mouse and human $(23,62)$. In our analyses we observed three genera from the family Ruminococcaceae that belonged to the top taxa found between PCOS and controls. None of the genera from Ruminococcaceae which we observed have previously been linked with PCOS, although the family itself and some its genera have been associated with PCOS in earlier studies $(25,26)$. It is possible that the different genera from Ruminococcaceae have similar metabolic functions that might affect the development of PCOS and its traits. Interestingly, in our study one particular genus, the Ruminococcaceae UCG-002, was positively associated with higher SHBG level, Matsuda and disposition indices. Finally, using 
a modelling-based analysis, we were able to identify two groups of microbial taxa whose relative abundance differentiate between PCOS and non-PCOS women. Although our data show modest discrimination between the PCOS and healthy women among the identified microbial groups, this analysis provides an important indication for future prediction studies.

PCOS is associated with metabolic derangements; more than half of the women are overweight or obese and PCOS raises a woman's risk of developing T2D and metabolic syndrome (63). In our cohort of 102 PCOS women, 38 were overweight and 27 were obese. Normal weight, overweight and obese PCOS individuals showed no statistically significant changes in bacterial diversities or in taxonomical composition between the three groups. Many earlier studies have shown that BMI has a crucial role in the diversity of gut microbiome and that higher BMI is strongly associated with gut dysbiosis (64-66). The reason for the observed inconsistency could have been the small sample size in our BMI based analyses in the PCOS group, as significant associations between diversity and BMI were observed when the entire cohort was analyzed. However, we were able to identify significant changes in both alpha and beta diversity when comparing women with PCOS classified based on OGTT as either NGT or preT2D. This is consistent with previous work, showing decreased richness and diversity in individuals with preT2D and T2D $(51,67)$. In addition, PCOS women with preT2D also showed higher abundance of genus Dorea compared to women in the NGT group. In a previous work, the genus Dorea has been linked to BMI and plasma metabolites such as glutamate and branched chain amino acids (BCAAs) which can predict BMI values (68). Naderpoor et al. showed, that the abundance of Dorea was positively correlated with fasting blood glucose (69). Interestingly, the abundance of Dorea was also significantly associated with various metabolic traits, such as BMI, glucose and insulin levels in our cohort [Supplementary Fig 6A-F (33)], providing additional support for the role of this genus in metabolic disorders. IR is common in women with PCOS 
although it is not included in diagnostic criteria for PCOS $(4,5)$. Cani et al. reported in their work that some Gram-negative bacteria have the ability to produce lipopolysaccharides which induce inflammation, IR, and obesity, all widely known traits of PCOS (70). Therefore, it is plausible that the gut microbiome is involved in the pathogenesis of PCOS by mediating IR and systemic inflammation. Moreover, the effect of microbiome could also be mediated through metabolites produced by gut microbiome. For example, in a study in mice, it was shown that the animals with diet-induced obesity showed improved insulin sensitivity after short chain fatty acid (SCFA) supplementation (71). SCFAs are produced through fermentation of non-digestible dietary fibers by the gut microbiome and they are an important energy source to the gut as well as performing as signaling molecules to affect the host metabolism (72). Therefore, it is possible that the dysbiosis of the gut that affects SCFA production is in turn, at least to some extent, responsible for the IR seen in PCOS individuals.

The strength of our current study is that the cohort was homogenous, all women belonging to the unique NFBC1966 with minimal ethic and geographic variation. PCOS cases and controls were all BMI-matched, thus the BMI effects on the comparisons between the cases and the controls are minimal. Furthermore, to our knowledge, the present study includes the largest number of PCOS women to date to investigate the associations between PCOS and the gut microbiome (73). Additionally, this is the first study assessing microbiota of women with PCOS in late fertile age.

Multiple reasons might explain why we could not confirm previously reported associations between microbiome and PCOS. The gut microbiome variation is affected by several factors such as diet, geography, medications, age, which all could possibly influence the results. In addition, the use of methods for sample collection, DNA extraction, 16S rRNA gene sequencing, and data analysis varies widely from study to study. Also, the use of small 
sample size in some previous studies may limit the ability to detect changes in specific bacteria. All of the above factors probably contributed to the discrepancies observed, but the identical age and large sample set are perhaps the most important factors that differed from previous studies. Earlier studies have been mainly focused on women of reproductive age, but the effects of the microbiome on the health of older women who are close to starting menopause might be different. At age 46, women with PCOS are less hyperandrogenic and metabolically more similar to non-PCOS women, who also have gained weight while ageing. It may be that at early and mid-reproductive years, PCOS women may more substantially differ from controls in the gut microbiome. Potential limitations of our study are selfreporting of PCOS symptoms and lack of ultrasonography. Women reporting in the 46-year questionnaire as having been diagnosed with PCO-ovaries on ultrasound represent possibly a milder hormonal and metabolic profile than the women diagnosed with PCOS in infertility clinics. Our study may therefore have underestimated the differences between PCOS and controls. However, the validity of self-reported PCOS phenotype has been well established $(31,32,34)$ and also supported by recently published data (74) and genetic analysis (75). Furthermore, in the case of PCOS, it would be interesting to investigate the microbiome not only of the gut but also from the reproductive tract. It is possible that PCOS-related infertility problems might also be associated with microbiome status as suggested by some early data (76). 


\section{CONCLUSIONS}

This study, utilizing the unique NFBC1966 cohort, revealed that women with PCOS in their late reproductive years have no large-scale difference in gut microbiome signature compared to age- and BMI-matched women. However, we did identify a number of differences between the microbial diversity, as well as specific taxa, and PCOS-related hormones and metabolic traits. In addition, we show clear differences in microbiome profile in PCOS women in a pre-diabetic state compared to PCOS women with normal glucose tolerance. Further (metagenomic, metabolomics, and functional) studies would be required to clarify the link between the gut microbiome, metabolites and development of PCOS. 


\section{ACKNOWLEDGEMENTS}

We thank all cohort members and researchers who participated in the 31 and $46 \mathrm{yrs}$ study. We also wish acknowledge the work of the NFBC project center.

\section{DECLARATIONS}

Ethics approval and consent to participate. All subjects have given written informed consent and the study was approved by the Ethics Committee of the Northern Ostrobothnia hospital district and was in accordance with the Helsinki Declaration.

Data availability. Individual-level 16S RNA sequencing data are submitted in the Sequence Read Archive (SRA) under the reference PRJNA669650. NFBC data is available from the University of Oulu, Infrastructure for Population Studies. Permission to use the data can be applied for research purposes via electronic material request portal. In the use of data, we follow the EU general data protection regulation (679/2016) and Finnish Data Protection Act. The use of personal data is based on cohort participant's written informed consent at his/her latest follow-up study, which may cause limitations to its use. Please, contact NFBC project center (NFBCprojectcenter@oulu.fi) and visit the cohort website (www.oulu.fi/nfbc) for more information.

Competing interest. The authors declare that they have no competing interests.

Authors' contributions. TTP, LMP, JST, SF, AS, SA and EO supervised the study. RKA, TTP and KHH oversaw collection of samples. ASL, NMM, JPD and SA prepared microbiome sequencing analysis. KL performed the data analysis with assistance of OA. KL, SA, RKA and EO prepared the manuscript with comments from other authors. 


\section{REFERENCES}

1. March WA, Moore VM, Willson KJ, Phillips DIW, Norman RJ, Davies MJ. The prevalence of polycystic ovary syndrome in a community sample assessed under contrasting diagnostic criteria. Hum. Reprod. 2010;25(2):544-551.

2. Teede HJ, Misso ML, Costello MF, Dokras A, Laven J, Moran L, Piltonen T, Norman RJ, Network IP. Recommendations from the international evidence-based guideline for the assessment and management of polycystic ovary syndrome. Hum. Reprod. 2018;33(9):1602-1618.

3. Zeng X, Xie Y-J, Liu Y-T, Long S-L, Mo Z-C. Polycystic ovarian syndrome: Correlation between hyperandrogenism, insulin resistance and obesity. Clin. Chim. Acta 2020;502:214-221.

4. Chen L, Xu WM, Zhang D. Association of abdominal obesity, insulin resistance, and oxidative stress in adipose tissue in women with polycystic ovary syndrome. Fertil. Steril. 2014;102(4):1167-1174.e4.

5. Luque-Ramírez M, Escobar-Morreale HF. Polycystic ovary syndrome as a paradigm for prehypertension, prediabetes, and preobesity. Curr. Hypertens. Rep. 2014;16(12):500.

6. Tremellen K, Pearce K. Dysbiosis of Gut Microbiota (DOGMA) - A novel theory for the development of Polycystic Ovarian Syndrome. Med. Hypotheses 2012;79(1):104112.

7. Coyte KZ, Schluter J, Foster KR. The ecology of the microbiome: Networks, competition, and stability. Science (80-. ). 2015;350(6261):663-666. 
8. Arumugam M, Raes J, Pelletier E, Le Paslier D, Yamada T, Mende DR, Fernandes GR, Tap J, Bruls T, Batto J-M, Bertalan M, Borruel N, Casellas F, Fernandez L, Gautier L, Hansen T, Hattori M, Hayashi T, Kleerebezem M, Kurokawa K, Leclerc M, Levenez F, Manichanh C, Nielsen HB, Nielsen T, Pons N, Poulain J, Qin J, Sicheritz-Ponten T, Tims S, Torrents D, Ugarte E, Zoetendal EG, Wang J, Guarner F, Pedersen O, de Vos WM, Brunak S, Doré J, Consortium M, Antolín M, Artiguenave F, Blottiere HM, Almeida M, Brechot C, Cara C, Chervaux C, Cultrone A, Delorme C, Denariaz G, Dervyn R, Foerstner KU, Friss C, van de Guchte M, Guedon E, Haimet F, Huber W, van Hylckama-Vlieg J, Jamet A, Juste C, Kaci G, Knol J, Lakhdari O, Layec S, Le Roux K, Maguin E, Mérieux A, Melo Minardi R, M'rini C, Muller J, Oozeer R, Parkhill J, Renault P, Rescigno M, Sanchez N, Sunagawa S, Torrejon A, Turner K, Vandemeulebrouck G, Varela E, Winogradsky Y, Zeller G, Weissenbach J, Ehrlich SD, Bork P. Enterotypes of the human gut microbiome. Nature 2011;473(7346):174-180.

9. Sonnenburg JL, Bäckhed F. Diet-microbiota interactions as moderators of human metabolism. Nature 2016;535(7610):56-64.

10. Allin KH, Tremaroli V, Caesar R, Jensen BAH, Damgaard MTF, Bahl MI, Licht TR, Hansen TH, Nielsen T, Dantoft TM, Linneberg A, Jørgensen T, Vestergaard H, Kristiansen K, Franks PW, Hansen T, Bäckhed F, Pedersen O. Aberrant intestinal microbiota in individuals with prediabetes. Diabetologia 2018;61(4):810 820.

11. Forslund K, Hildebrand F, Nielsen T, Falony G, Le Chatelier E, Sunagawa S, Prifti E, Vieira-Silva S, Gudmundsdottir V, Krogh Pedersen H, Arumugam M, Kristiansen K, Voigt AY, Vestergaard H, Hercog R, Igor Costea P, Kultima JR, Li J, Jorgensen T, Levenez F, Dore J, Nielsen HB, Brunak S, Raes J, Hansen T, Wang J, Ehrlich SD, Bork P, Pedersen O. Disentangling type 2 diabetes and metformin treatment signatures in the human gut microbiota. Nature 2015;528(7581):262-266.

12. Karlsson FH, Tremaroli V, Nookaew I, Bergström G, Behre CJ, Fagerberg B, Nielsen J, Bäckhed F. Gut metagenome in European women with normal, impaired and diabetic glucose control. Nature 2013;498(7452):99-103. 
13. Larsen N, Vogensen FK, Van Den Berg FWJ, Nielsen DS, Andreasen AS, Pedersen BK, Al-Soud WA, Sørensen SJ, Hansen LH, Jakobsen M. Gut microbiota in human adults with type 2 diabetes differs from non-diabetic adults. PLoS One 2010;5(2). doi:10.1371/journal.pone.0009085.

14. Bäckhed F, Ding H, Wang T, Hooper L V, Koh GY, Nagy A, Semenkovich CF, Gordon JI. The gut microbiota as an environmental factor that regulates fat storage. Proc. Natl. Acad. Sci. U. S. A. 2004;101(44):15718-15723.

15. Haro C, García-Carpintero S, Rangel-Zúñiga OA, Alcalá-Díaz JF, Landa BB, Clemente JC, Pérez-Martínez P, López-Miranda J, Pérez-Jiménez F, Camargo A. Consumption of Two Healthy Dietary Patterns Restored Microbiota Dysbiosis in Obese Patients with Metabolic Dysfunction. Mol. Nutr. Food Res. 2017;61(12). doi:10.1002/mnfr.201700300.

16. Turnbaugh PJ, Ley RE, Mahowald MA, Magrini V, Mardis ER, Gordon JI. An obesity-associated gut microbiome with increased capacity for energy harvest. Nature 2006;444(7122):1027-1031.

17. Loftfield E, Herzig K-H, Caporaso JG, Derkach A, Wan Y, Byrd DA, Vogtmann E, Männikkö M, Karhunen V, Knight R, Gunter MJ, Järvelin M-R, Sinha R. Association of Body Mass Index with Fecal Microbial Diversity and Metabolites in the Northern Finland Birth Cohort. Cancer Epidemiol. Biomarkers \&amp;amp; Prev. 2020. doi:10.1158/1055-9965.EPI-20-0824.

18. Pedersen HK, Gudmundsdottir V, Nielsen HB, Hyotylainen T, Nielsen T, Jensen BAH, Forslund K, Hildebrand F, Prifti E, Falony G, Le Chatelier E, Levenez F, Doré J, Mattila I, Plichta DR, Pöhö P, Hellgren LI, Arumugam M, Sunagawa S, Vieira-Silva S, Jørgensen T, Holm JB, Trošt K, Consortium M, Kristiansen K, Brix S, Raes J, Wang J, Hansen T, Bork P, Brunak S, Oresic M, Ehrlich SD, Pedersen O. Human gut microbes impact host serum metabolome and insulin sensitivity. Nature 2016;535(7612):376-381.

19. Aron-Wisnewsky J, Prifti E, Belda E, Ichou F, Kayser BD, Dao MC, Verger EO, Hedjazi L, Bouillot J-L, Chevallier J-M, Pons N, Le Chatelier E, Levenez F, Ehrlich SD, Dore J, Zucker J-D, Clément K. Major microbiota dysbiosis in severe obesity: fate after bariatric surgery. Gut 2019;68(1):70-82. 
20. Luna RA, Foster JA. Gut brain axis: diet microbiota interactions and implications for modulation of anxiety and depression. Curr. Opin. Biotechnol. 2015;32:35-41.

21. Schachter J, Martel J, Lin C-S, Chang C-J, Wu T-R, Lu C-C, Ko Y-F, Lai H-C, Ojcius DM, Young JD. Effects of obesity on depression: A role for inflammation and the gut microbiota. Brain Behav. Immun. 2018;69:1-8.

22. Valles-Colomer M, Falony G, Darzi Y, Tigchelaar EF, Wang J, Tito RY, Schiweck C, Kurilshikov A, Joossens M, Wijmenga C, Claes S, Van Oudenhove L, Zhernakova A, Vieira-Silva S, Raes J. The neuroactive potential of the human gut microbiota in quality of life and depression. Nat Microbiol 2019;4(4):623-632.

23. Insenser M, Murri M, Del Campo R, Martínez-García MÁ, Fernández-Durán E, Escobar-Morreale HF. Gut Microbiota and the Polycystic Ovary Syndrome: Influence of Sex, Sex Hormones, and Obesity. J. Clin. Endocrinol. Metab. 2018;103(7):2552-2562.

24. Lindheim L, Bashir M, Münzker J, Trummer C, Zachhuber V, Leber B, Horvath A, Pieber TR, Gorkiewicz G, Stadlbauer V, Obermayer-Pietsch B. Alterations in Gut Microbiome Composition and Barrier Function Are Associated with Reproductive and Metabolic Defects in Women with Polycystic Ovary Syndrome (PCOS): A Pilot Study. PLoS One 2017;12(1):e0168390.

25. Liu R, Zhang C, Shi Y, Zhang F, Li L, Wang X, Ling Y, Fu H, Dong W, Shen J, Reeves A, Greenberg AS, Zhao L, Peng Y, Ding X. Dysbiosis of Gut Microbiota Associated with Clinical Parameters in Polycystic Ovary Syndrome. Front. Microbiol. $2017 ; 8: 324$.

26. Torres PJ, Siakowska M, Banaszewska B, Pawelczyk L, Duleba AJ, Kelley ST, Thackray VG. Gut Microbial Diversity in Women With Polycystic Ovary Syndrome Correlates With Hyperandrogenism. J. Clin. Endocrinol. Metab. 2018;103(4):15021511.

27. Zhou L, Ni Z, Cheng W, Yu J, Sun S, Zhai D, Yu C, Cai Z. Characteristic gut microbiota and predicted metabolic functions in women with PCOS. Endocr Connect 2020;9(1):63-73.

28. Rantakallio P. The longitudinal study of the northern Finland birth cohort of 1966. 
Paediatr. Perinat. Epidemiol. 1988;2(1):59-88.

29. University of Oulu, Northern Finland Birth Cohort 1966. Available at: https://etsin.fairdata.fi/dataset/716939c3-7a2a-4b6a-91f3-92aca09bc52d

30. Karjula S, Morin-Papunen L, Auvinen J, Ruokonen A, Puukka K, Franks S, Järvelin M-R, Tapanainen JS, Jokelainen J, Miettunen J, Piltonen TT. Psychological Distress Is More Prevalent in Fertile Age and Premenopausal Women With PCOS Symptoms: 15-Year Follow-Up. J. Clin. Endocrinol. Metab. 2017;102(6):1861-1869.

31. Koivuaho E, Laru J, Ojaniemi M, Puukka K, Kettunen J, Tapanainen JS, Franks S, Järvelin M-R, Morin-Papunen L, Sebert S, Piltonen TT. Age at adiposity rebound in childhood is associated with PCOS diagnosis and obesity in adulthoodlongitudinal analysis of BMI data from birth to age 46 in cases of PCOS. Int. J. Obes. 2019;43(7):1370-1379.

32. Ollila M-ME, Piltonen T, Puukka K, Ruokonen A, Järvelin M-R, Tapanainen JS, Franks S, Morin-Papunen L. Weight Gain and Dyslipidemia in Early Adulthood Associate With Polycystic Ovary Syndrome: Prospective Cohort Study. J. Clin. Endocrinol. Metab. 2016;101(2):739-747.

33. Lüll K, Arffmann RK, Sola-Leyva A, Molina NM, Aasmets O, Herzig K-H, PlazaDíaz J, Franks S, Morin-Papunen L, Tapanainen JS, Salumets A, Altmäe S, Piltonen TT, Org E. Data from: The Gut Microbiome in Polycystic Ovary Syndrome and its Association with Metabolic Traits. Figshare 2020. doi:10.6084/m9.figshare.12993047. Deposited 24 September 2020.

34. Taponen S, Ahonkallio S, Martikainen H, Koivunen R, Ruokonen A, Sovio U, Hartikainen A-L, Pouta A, Laitinen J, King V, Franks S, McCarthy MI, Järvelin M-R. Prevalence of polycystic ovaries in women with self-reported symptoms of oligomenorrhoea and/or hirsutism: Northern Finland Birth Cohort 1966 Study. Hum. Reprod. 2004;19(5):1083-1088.

35. Matsuda M, DeFronzo RA. Insulin sensitivity indices obtained from oral glucose tolerance testing: comparison with the euglycemic insulin clamp. Diabetes Care 1999;22(9):1462 LP - 1470. 
36. WHO. Definition and diagnosis of diabetes mellitus and intermediate hyperglycaemia. 2006;ISBN: 978.

37. Bolyen E, Rideout JR, Dillon MR, Bokulich NA, Abnet CC, Al-Ghalith GA, Alexander H, Alm EJ, Arumugam M, Asnicar F, Bai Y, Bisanz JE, Bittinger K, Brejnrod A, Brislawn CJ, Brown CT, Callahan BJ, Caraballo-Rodríguez AM, Chase J, Cope EK, Da Silva R, Diener C, Dorrestein PC, Douglas GM, Durall DM, Duvallet C, Edwardson CF, Ernst M, Estaki M, Fouquier J, Gauglitz JM, Gibbons SM, Gibson DL, Gonzalez A, Gorlick K, Guo J, Hillmann B, Holmes S, Holste H, Huttenhower C, Huttley GA, Janssen S, Jarmusch AK, Jiang L, Kaehler BD, Kang K Bin, Keefe CR, Keim P, Kelley ST, Knights D, Koester I, Kosciolek T, Kreps J, Langille MGI, Lee J, Ley R, Liu Y-X, Loftfield E, Lozupone C, Maher M, Marotz C, Martin BD, McDonald D, McIver LJ, Melnik A V, Metcalf JL, Morgan SC, Morton JT, Naimey AT, Navas-Molina JA, Nothias LF, Orchanian SB, Pearson T, Peoples SL, Petras D, Preuss ML, Pruesse E, Rasmussen LB, Rivers A, Robeson 2nd MS, Rosenthal P, Segata N, Shaffer M, Shiffer A, Sinha R, Song SJ, Spear JR, Swafford AD, Thompson LR, Torres PJ, Trinh P, Tripathi A, Turnbaugh PJ, Ul-Hasan S, van der Hooft JJJ, Vargas F, Vázquez-Baeza Y, Vogtmann E, von Hippel M, Walters W, Wan Y, Wang M, Warren J, Weber KC, Williamson CHD, Willis AD, Xu ZZ, Zaneveld JR, Zhang Y, Zhu Q, Knight R, Caporaso JG. Reproducible, interactive, scalable and extensible microbiome data science using QIIME 2. Nat. Biotechnol. 2019;37(8):852857.

38. Callahan BJ, McMurdie PJ, Rosen MJ, Han AW, Johnson AJA, Holmes SP. DADA2: High-resolution sample inference from Illumina amplicon data. Nat. Methods 2016;13(7):581-583.

39. Katoh K, Standley DM. MAFFT multiple sequence alignment software version 7: improvements in performance and usability. Mol. Biol. Evol. 2013;30(4):772-780.

40. Price MN, Dehal PS, Arkin AP. FastTree 2--approximately maximum-likelihood trees for large alignments. PLoS One 2010;5(3):e9490.

41. Bokulich NA, Kaehler BD, Rideout JR, Dillon M, Bolyen E, Knight R, Huttley GA, Gregory Caporaso J. Optimizing taxonomic classification of marker-gene 
amplicon sequences with QIIME 2's q2-feature-classifier plugin. Microbiome 2018;6(1). doi:10.1186/s40168-018-0470-z.

42. Quast C, Pruesse E, Yilmaz P, Gerken J, Schweer T, Yarza P, Peplies J, Glöckner FO. The SILVA ribosomal RNA gene database project: improved data processing and web-based tools. Nucleic Acids Res. 2013;41(Database issue):D590-6.

43. Gómez-Rubio V. ggplot2 - Elegant Graphics for Data Analysis (2nd Edition). J. Stat. Softw. 2017;77(Book Review 2). doi:10.18637/jss.v077.b02.

44. Wei T, Simko V. R package "corrplot": Visualization of a Correlation Matrix (Version 0.84). 2017. Available at: https://github.com/taiyun/corrplot.

45. Benjamini Y, Hochberg Y. Controlling the False Discovery Rate: A Practical and Powerful Approach to Multiple Testing. J. R. Stat. Soc. Ser. B 1995;57(1):289-300.

46. McMurdie PJ, Holmes S. phyloseq: An R Package for Reproducible Interactive Analysis and Graphics of Microbiome Census Data. PLoS One 2013;8(4):e61217.

47. Lahti L, Shetty S. Microbiome R package. Available at: url: http://microbiome.github.io.

48. Langfelder P, Horvath S. WGCNA: an R package for weighted correlation network analysis. BMC Bioinformatics 2008;9(1). doi:10.1186/1471-2105-9-559.

49. Fernandes AD, Macklaim JM, Linn TG, Reid G, Gloor GB. ANOVA-Like Differential Expression (ALDEx) Analysis for Mixed Population RNA-Seq. PLoS One 2013;8(7):e67019.

50. Rivera-Pinto J, Egozcue JJ, Pawlowsky-Glahn V, Paredes R, Noguera-Julian M, Calle ML. Balances: a New Perspective for Microbiome Analysis. mSystems 2018;3(4). doi:10.1128/mSystems.00053-18.

51. Rubin KH, Glintborg D, Nybo M, Abrahamsen B, Andersen M. Development and Risk Factors of Type 2 Diabetes in a Nationwide Population of Women With Polycystic Ovary Syndrome. J. Clin. Endocrinol. Metab. 2017;102(10):3848-3857.

52. Gurung M, Li Z, You H, Rodrigues R, Jump DB, Morgun A, Shulzhenko N. Role of gut microbiota in type 2 diabetes pathophysiology. EBioMedicine 2020;51:102590. 
53. Senghora B, Sokhnab C, Ruimycde R, Lagiera J-C. Gut microbiota diversity according to dietary habits and geographical provenance. Hum. Microbiome J. 2018;78(1):1-9.

54. Kurilshikov A, Medina-Gomez C, Bacigalupe R, Radjabzadeh D, Wang J, Demirkan A, Le Roy CI, Raygoza Garay JA, Finnicum CT, Liu X, Zhernakova D V, Bonder MJ, Hansen TH, Frost F, Rühlemann MC, Turpin W, Moon J-Y, Kim H-N, Lüll K, Barkan E, Shah SA, Fornage M, Szopinska-Tokov J, Wallen ZD, Borisevich D, Agreus L, Andreasson A, Bang C, Bedrani L, Bell JT, Bisgaard H, Boehnke M, Boomsma DI, Burk RD, Claringbould A, Croitoru K, Davies GE, van Duijn CM, Duijts L, Falony G, Fu J, van der Graaf A, Hansen T, Homuth G, Hughes DA, Ijzerman RG, Jackson MA, Jaddoe VW V, Joossens M, Jørgensen T, Keszthelyi D, Knight R, Laakso M, Laudes M, Launer LJ, Lieb W, Lusis AJ, Masclee AAM, Moll HA, Mujagic Z, Qibin Q, Rothschild D, Shin H, Sørensen SJ, Steves CJ, Thorsen J, Timpson NJ, Tito RY, Vieira-Silva S, Völker U, Völzke H, Võsa U, Wade KH, Walter S, Watanabe K, Weiss S, Weiss FU, Weissbrod O, Westra H-J, Willemsen G, Payami H, Jonkers DMAE, Vasquez AA, de Geus EJC, Meyer KA, Stokholm J, Segal E, Org E, Wijmenga C, Kim H-L, Kaplan RC, Spector TD, Uitterlinden AG, Rivadeneira F, Franke A, Lerch MM, Franke L, Sanna S, D’Amato M, Pedersen O, Paterson AD, Kraaij R, Raes J, Zhernakova A. Genetics of human gut microbiome composition. bioRxiv 2020:2020.06.26.173724.

55. Qi X, Yun C, Sun L, Xia J, Wu Q, Wang Y, Wang L, Zhang Y, Liang X, Wang L, Gonzalez FJ, Patterson AD, Liu H, Mu L, Zhou Z, Zhao Y, Li R, Liu P, Zhong C, Pang Y, Jiang C, Qiao J. Gut microbiota-bile acid-interleukin-22 axis orchestrates polycystic ovary syndrome. Nat. Med. 2019;25(8):1225-1233.

56. Haudum C, Lindheim L, Ascani A, Trummer C, Horvath A, Münzker J, Obermayer-Pietsch B. Impact of Short-Term Isoflavone Intervention in Polycystic Ovary Syndrome (PCOS) Patients on Microbiota Composition and Metagenomics. Nutrients 2020;12(6). doi:10.3390/nu12061622.

57. Chu W, Han Q, Xu J, Wang J, Sun Y, Li W, Chen Z-J, Du Y. Metagenomic analysis identified microbiome alterations and pathological association between intestinal microbiota and polycystic ovary syndrome. Fertil. Steril. 2020;113(6):1286- 
1298.e4.

58. Eyupoglu ND, Ergunay K, Acikgoz A, Akyon Y, Yilmaz E, Yildiz BO. Gut microbiota and oral contraceptive use in overweight and obese patients with polycystic ovary syndrome. J. Clin. Endocrinol. Metab. 2020. doi:10.1210/clinem/dgaa600.

59. Kasai C, Sugimoto K, Moritani I, Tanaka J, Oya Y, Inoue H, Tameda M, Shiraki K, Ito M, Takei Y, Takase K. Comparison of the gut microbiota composition between obese and non-obese individuals in a Japanese population, as analyzed by terminal restriction fragment length polymorphism and next-generation sequencing. BMC Gastroenterol. 2015;15(1). doi:10.1186/s12876-015-0330-2.

60. Moreno-Indias I, Sánchez-Alcoholado L, Sánchez-Garrido MÁ, Martín-Núñez GM, Pérez-Jiménez F, Tena-Sempere M, Tinahones FJ, Queipo-Ortuño MI. Neonatal Androgen Exposure Causes Persistent Gut Microbiota Dysbiosis Related to Metabolic Disease in Adult Female Rats. Endocrinology 2016;157(12):4888-4898.

61. Sherman SB, Sarsour N, Salehi M, Schroering A, Mell B, Joe B, Hill JW. Prenatal androgen exposure causes hypertension and gut microbiota dysbiosis. Gut Microbes 2018;9(5):400-421.

62. Kelley ST, Skarra D V, Rivera AJ, Thackray VG. The Gut Microbiome Is Altered in a Letrozole-Induced Mouse Model of Polycystic Ovary Syndrome. PLoS One 2016;11(1):e0146509.

63. Gambineri A, Patton L, Altieri P, Pagotto U, Pizzi C, Manzoli L, Pasquali R. Polycystic ovary syndrome is a risk factor for type 2 diabetes: results from a longterm prospective study. Diabetes 2012;61(9):2369-2374.

64. Barlow GM, Yu A, Mathur R. Role of the Gut Microbiome in Obesity and Diabetes Mellitus. Nutr. Clin. Pr. 2015;30(6):787-797.

65. Guirro M, Costa A, Gual-Grau A, Herrero P, Torrell H, Canela N, Arola L. Effects from diet-induced gut microbiota dysbiosis and obesity can be ameliorated by fecal microbiota transplantation: A multiomics approach. PLoS One 2019;14(9):e0218143.

66. Saad MJA, Santos A, Prada PO. Linking Gut Microbiota and Inflammation to 
Obesity and Insulin Resistance. Physiol. 2016;31(4):283-293.

67. Li Q, Chang Y, Zhang K, Chen H, Tao S, Zhang Z. Implication of the gut microbiome composition of type 2 diabetic patients from northern China. Sci. Rep. 2020;10(1):5450.

68. Ottosson F, Brunkwall L, Ericson U, Nilsson PM, Almgren P, Fernandez C, Melander O, Orho-Melander M. Connection Between BMI-Related Plasma Metabolite Profile and Gut Microbiota. J. Clin. Endocrinol. Metab. 2018;103(4):14911501.

69. Naderpoor N, Mousa A, Gomez-Arango L, Barrett H, Nitert MD, de Courten B. Faecal Microbiota Are Related to Insulin Sensitivity and Secretion in Overweight or Obese Adults. J. Clin. Med. 2019;8(4):452.

70. Cani PD, Amar J, Iglesias MA, Poggi M, Knauf C, Bastelica D, Neyrinck AM, Fava F, Tuohy KM, Chabo C, Waget A, Delmée E, Cousin B, Sulpice T, Chamontin B, Ferrières J, Tanti J-F, Gibson GR, Casteilla L, Delzenne NM, Alessi MC, Burcelin R. Metabolic endotoxemia initiates obesity and insulin resistance. Diabetes 2007;56(7):1761-1772.

71. Perry RJ, Peng L, Barry NA, Cline GW, Zhang D, Cardone RL, Petersen KF, Kibbey RG, Goodman AL, Shulman GI. Acetate mediates a microbiome-brain- $\beta$ cell axis to promote metabolic syndrome. Nature 2016;534(7606):213-217.

72. Flint HJ, Scott KP, Duncan SH, Louis P, Forano E. Microbial degradation of complex carbohydrates in the gut. Gut Microbes 2012;3(4):289-306.

73. Thackray VG. Sex, Microbes, and Polycystic Ovary Syndrome. Trends Endocrinol. Metab. 2019;30(1):54-65.

74. Chan JL, Pall M, Ezeh U, Mathur R, Pisarska MD, Azziz R. Screening for Androgen Excess in Women: Accuracy of Self-Reported Excess Body Hair Growth and Menstrual Dysfunction. J. Clin. Endocrinol. Metab. 2020;105(10). doi:10.1210/clinem/dgz264.

75. Day F, Karaderi T, Jones MR, Meun C, He C, Drong A, Kraft P, Lin N, Huang H, Broer L, Magi R, Saxena R, Laisk T, Urbanek M, Hayes MG, Thorleifsson G, 
Fernandez-Tajes J, Mahajan A, Mullin BH, Stuckey BGA, Spector TD, Wilson SG, Goodarzi MO, Davis L, Obermayer-Pietsch B, Uitterlinden AG, Anttila V, Neale BM, Jarvelin M-R, Fauser B, Kowalska I, Visser JA, Andersen M, Ong K, Stener-Victorin E, Ehrmann D, Legro RS, Salumets A, McCarthy MI, MorinPapunen L, Thorsteinsdottir U, Stefansson K, Team 23andMe Research, Styrkarsdottir U, Perry JRB, Dunaif A, Laven J, Franks S, Lindgren CM, Welt CK. Large-scale genome-wide meta-analysis of polycystic ovary syndrome suggests shared genetic architecture for different diagnosis criteria. PLoS Genet. 2018;14(12):e1007813-e1007813.

76. Moreno I, Simon C. Relevance of assessing the uterine microbiota in infertility. Fertil. Steril. 2018;110(3):337-343. 


\section{FIGURES}

Figure 1: Landscape of microbiome composition in the entire cohort. (A) Pie chart indicates the average relative abundances of the top major phyla in the cohort. (B) Mean relative abundance of the core microbiome (taxa present in over 95\% of individuals) of the cohort at genus level.

Figure 2: Comparison of the gut microbiome diversity between control and PCOS samples. (A) Box-plots of the Shannon diversity index (alpha diversity) $(\mathrm{p}=0.979)$, median values and interquartile ranges have been indicated on the plots. Beta diversity is represented by Principal Coordinate Analysis $(\mathrm{PCoA})$ based on $(\mathrm{B})$ unweighted UniFrac $(\mathrm{p}=0.175)$ and $(\mathrm{C})$ weighted UniFrac $(\mathrm{p}=0.44)$ distances. Selbal analysis results $(\mathrm{D})$. The balance is made out of two groups of taxa: Paraprevotella-Streptococcus and Eubacterium ventriosum groupBifidobacterium. The boxplots characterize the distribution of the balance scores for PCOS women and healthy controls. Each plot point on panels $\mathrm{B}$ and $\mathrm{C}$ represents a single individual, the shapes indicate study groups (rhombus: controls, star: PCOS). The right part of the panel D holds the ROC curve with its AUC value (0.643), and density curve. Abbreviations: AUC: area under the curve; FPR: false positive rate; PCOS: polycystic ovary syndrome; ROC: receiver operating characteristic curve; TPR: true positive rate.

Figure 3: Correlation heatmap of hormonal and metabolic biomarkers in the entire cohort at the age 46, and involving top 10 bacteria associated with differences between PCOS and control women. Key: $*$ FDR $\leq 0.2 ; * *$ FDR $\leq 0.1$. The color key indicates the correlation direction (blue: negative correlation, red: positive correlation). Abbreviations: BMI: body mass index; FDR: false discovery rate; Hb1Ac: glycated haemoglobin; SHGB: sex hormone binding globulin. 
Figure 4: Comparison of the gut microbiome diversity between NGT $(n=76)$ and preT2D samples $(n=14)$ among women with PCOS. (A) Box-plots of the Shannon diversity Index (alpha diversity), median values and interquartile ranges have been indicated in the plot ( $\mathrm{p}=$ 0.018). (B) Beta diversity is represented by Principal Coordinate Analysis (PCoA) based on unweighted UniFrac distance $(\mathrm{p}=0.003)$. (C) Genus Dorea with statistically significant differences $(\mathrm{FDR}=0.03)$. NGT: normal glucose tolerance; preT2D: pre-type 2 diabetes. Each plot point represents a single individual, the shapes indicate study groups (rhombus: NGT, star: preT2D). 
Table 1. Clinical, metabolic, and hormonal characteristics of study population.

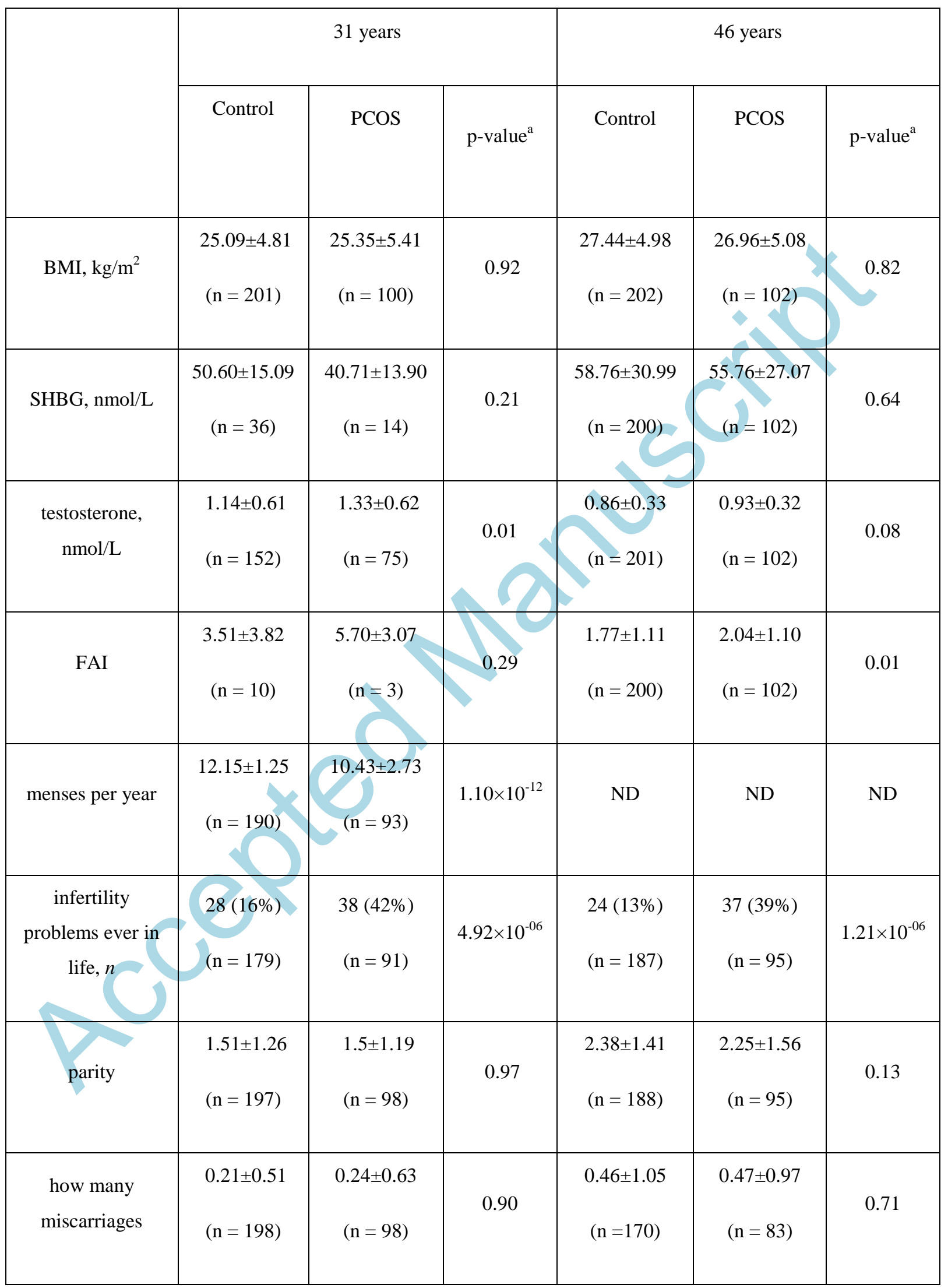




\begin{tabular}{|c|c|c|c|c|c|c|}
\hline $\begin{array}{c}\text { fasting glucose, } \\
\mathrm{mmol} / \mathrm{L}\end{array}$ & $\begin{array}{l}4.96 \pm 0.49 \\
(n=156)\end{array}$ & $\begin{array}{l}5.15 \pm 1.24 \\
(n=78)\end{array}$ & 0.19 & $\begin{array}{l}5.05 \pm 0.92 \\
(n=195)\end{array}$ & $\begin{array}{c}5.38 \pm 0.58 \\
(n=95)\end{array}$ & 0.55 \\
\hline $\begin{array}{c}2 \mathrm{~h} \text { glucose, } \\
\mathrm{mmol} / \mathrm{L}\end{array}$ & ND & ND & ND & $\begin{array}{l}5.88 \pm 1.63 \\
(n=193)\end{array}$ & $\begin{array}{c}5.69 \pm 1.45 \\
(n=92)\end{array}$ & 0.24 \\
\hline $\begin{array}{c}\text { fasting insulin, } \\
\mathrm{mU} / \mathrm{L}\end{array}$ & $\begin{array}{l}8.52 \pm 3.79 \\
(n=155)\end{array}$ & $\begin{array}{c}9.14 \pm 4.99 \\
(n=78)\end{array}$ & 0.70 & $\begin{array}{l}9.82 \pm 5.65 \\
(n=195)\end{array}$ & $\begin{array}{c}10.2 \pm 7.63 \\
(\mathrm{n}=95)\end{array}$ & 0.79 \\
\hline $2 \mathrm{~h}$ insulin, $\mathrm{mU} / \mathrm{L}$ & ND & ND & ND & $\begin{array}{c}63.03 \pm 50.61 \\
(n=193)\end{array}$ & $\begin{array}{c}57.34 \pm 41.81 \\
(n=93)\end{array}$ & 0.62 \\
\hline Matsuda index & ND & ND & ND & $4.84 \pm 2.55$ & $\begin{array}{c}5.24 \pm 3.35 \\
(n-89)\end{array}$ & 0.77 \\
\hline disposition index & ND & ND & ND & $\begin{array}{c}186.15 \pm 87.32 \\
(n=190)\end{array}$ & $\begin{array}{c}189.47 \pm 89.59 \\
(\mathrm{n}=89)\end{array}$ & 0.74 \\
\hline
\end{tabular}

Data are presented as mean \pm standard deviation for continuous traits and as absolute proportions and prevalence (\%). ${ }^{\text {a } W i l c o x o n ~ s i g n ~ r a n k ~ t e s t ~(c o n t i n u o u s ~ v a r i a b l e) ~ o r ~ F i s h e r ' s ~ E x a c t ~ t e s t ~(c a t e g o r i c a l ~ v a r i a b l e) . ~ T h e ~}$ number of women in separate analyses varies due to non-response to some items. Abbreviations: BMI: body mass index; FAI: Free Androgen Index; $n$ : number of individuals; ND: no data; SHBG: sex hormone binding globulin; y: year. 


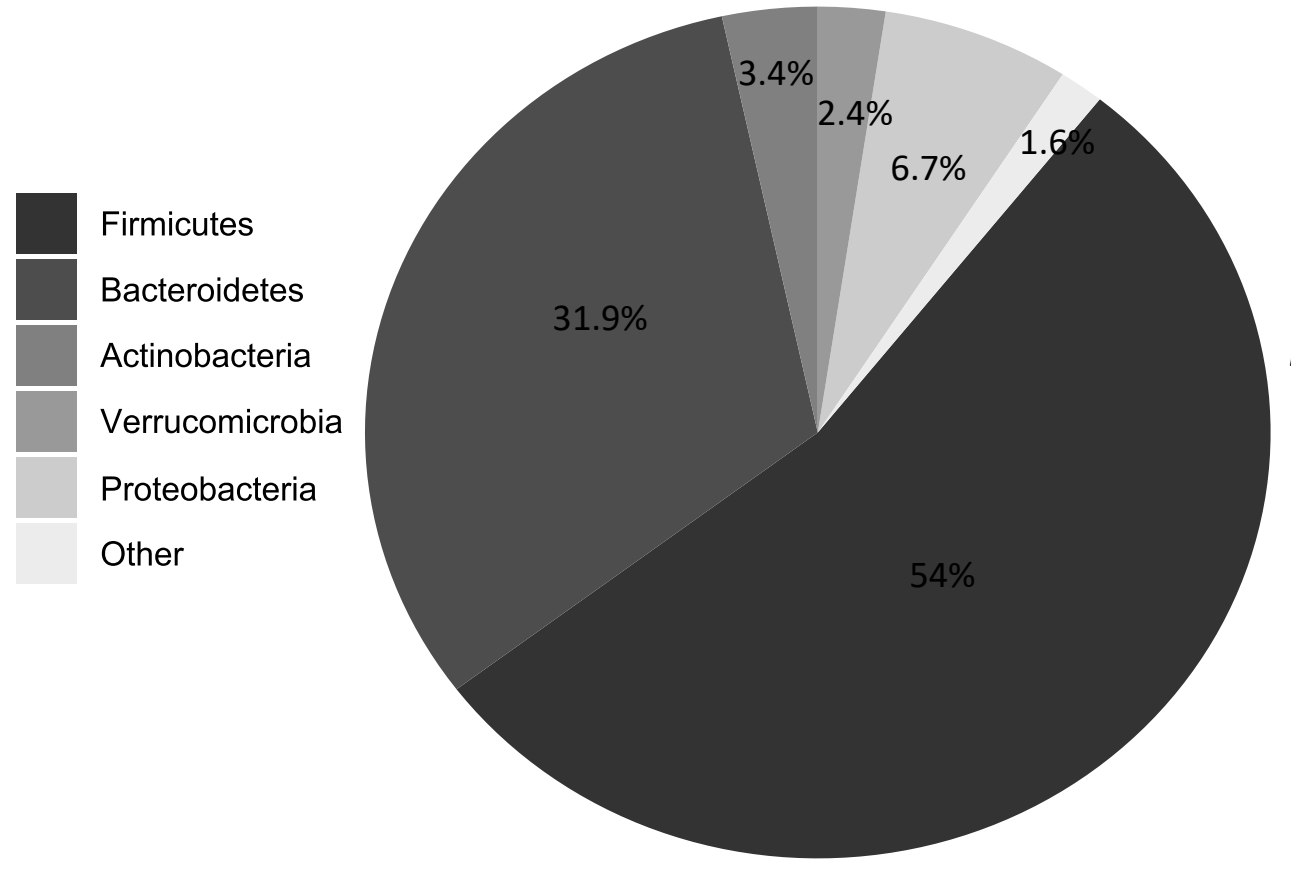

Ruminococcaceae uncultured

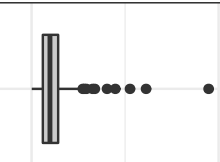

追 Bacteroidetes

追 Firmicutes





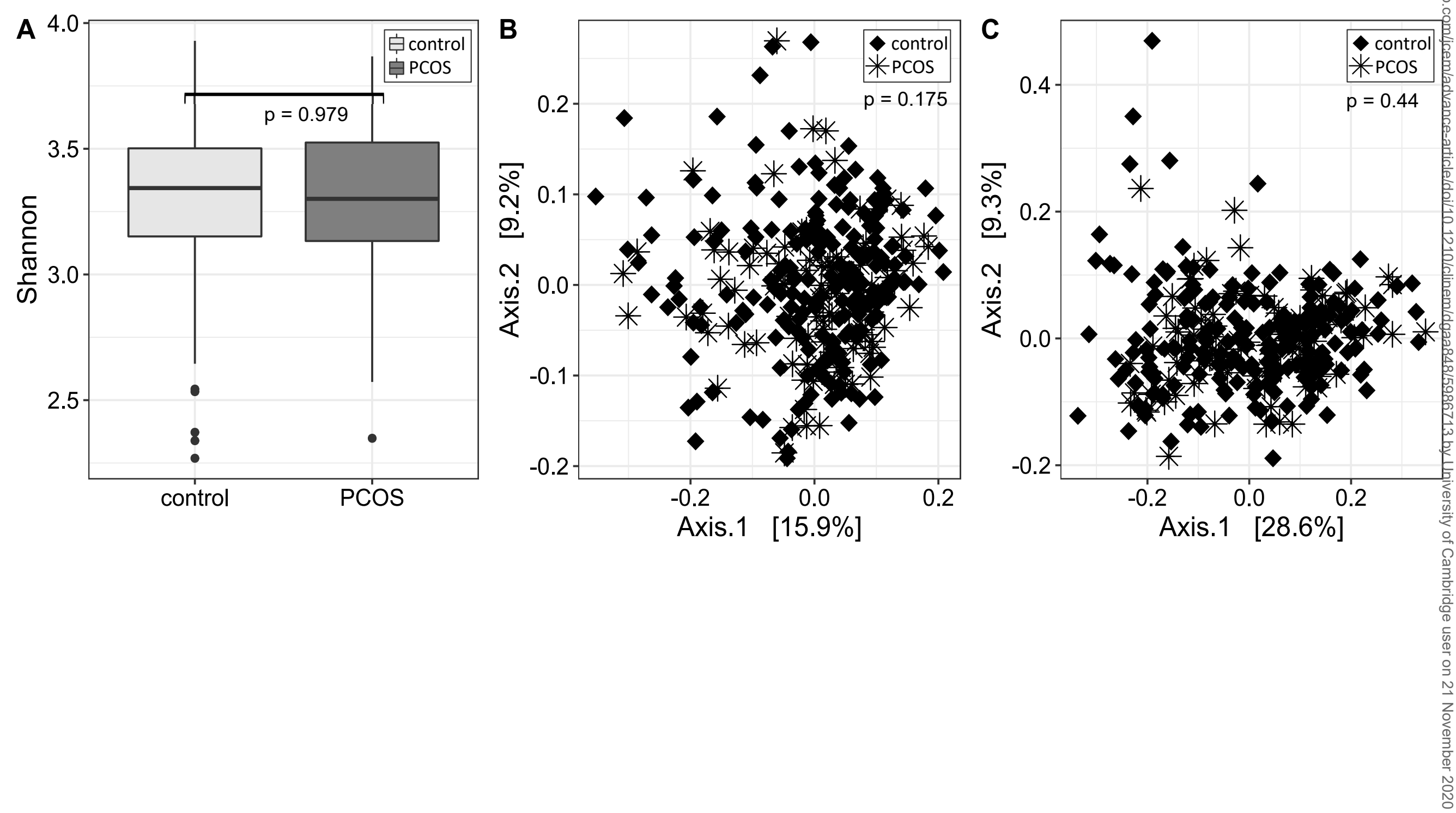
D
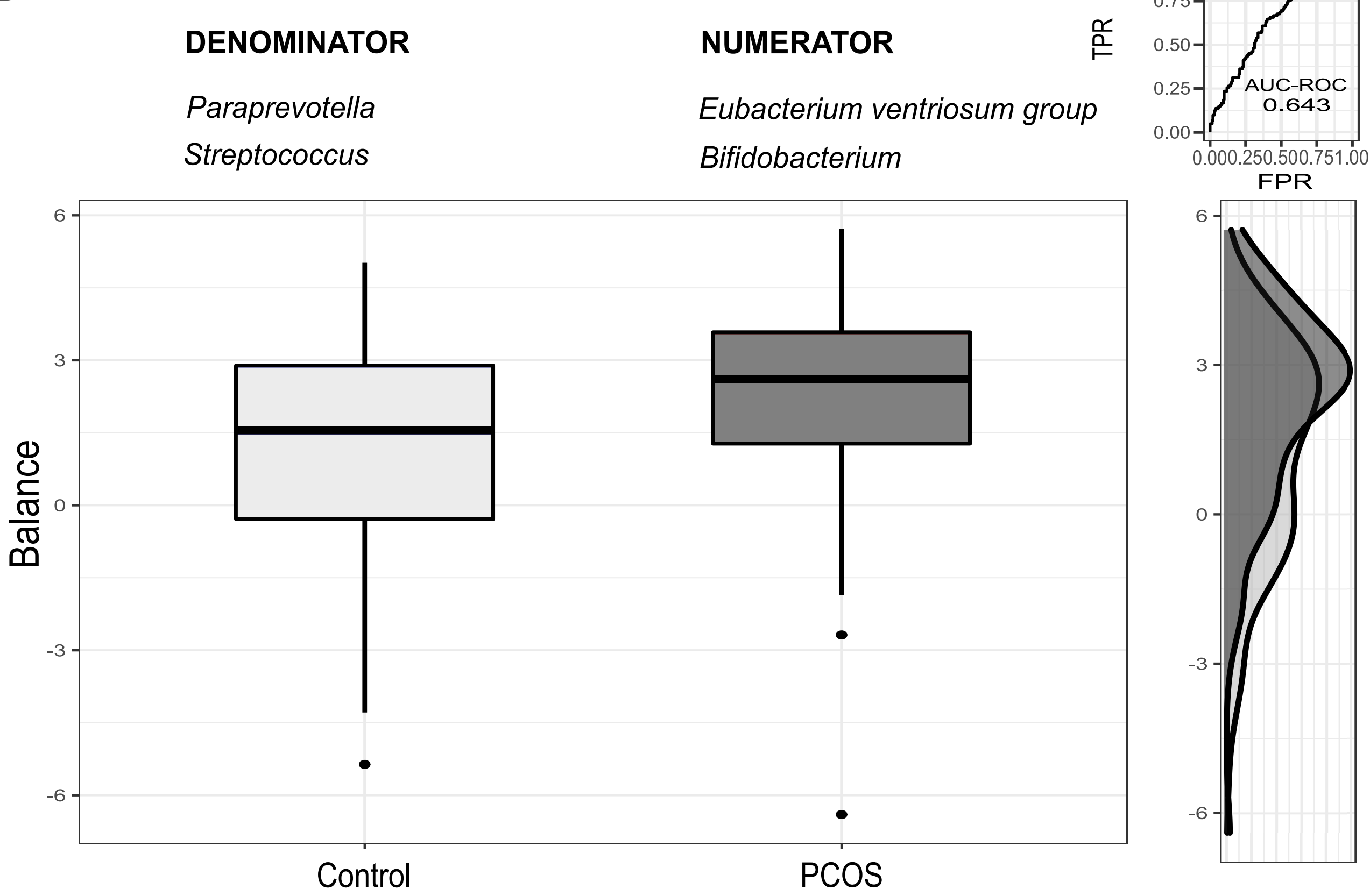


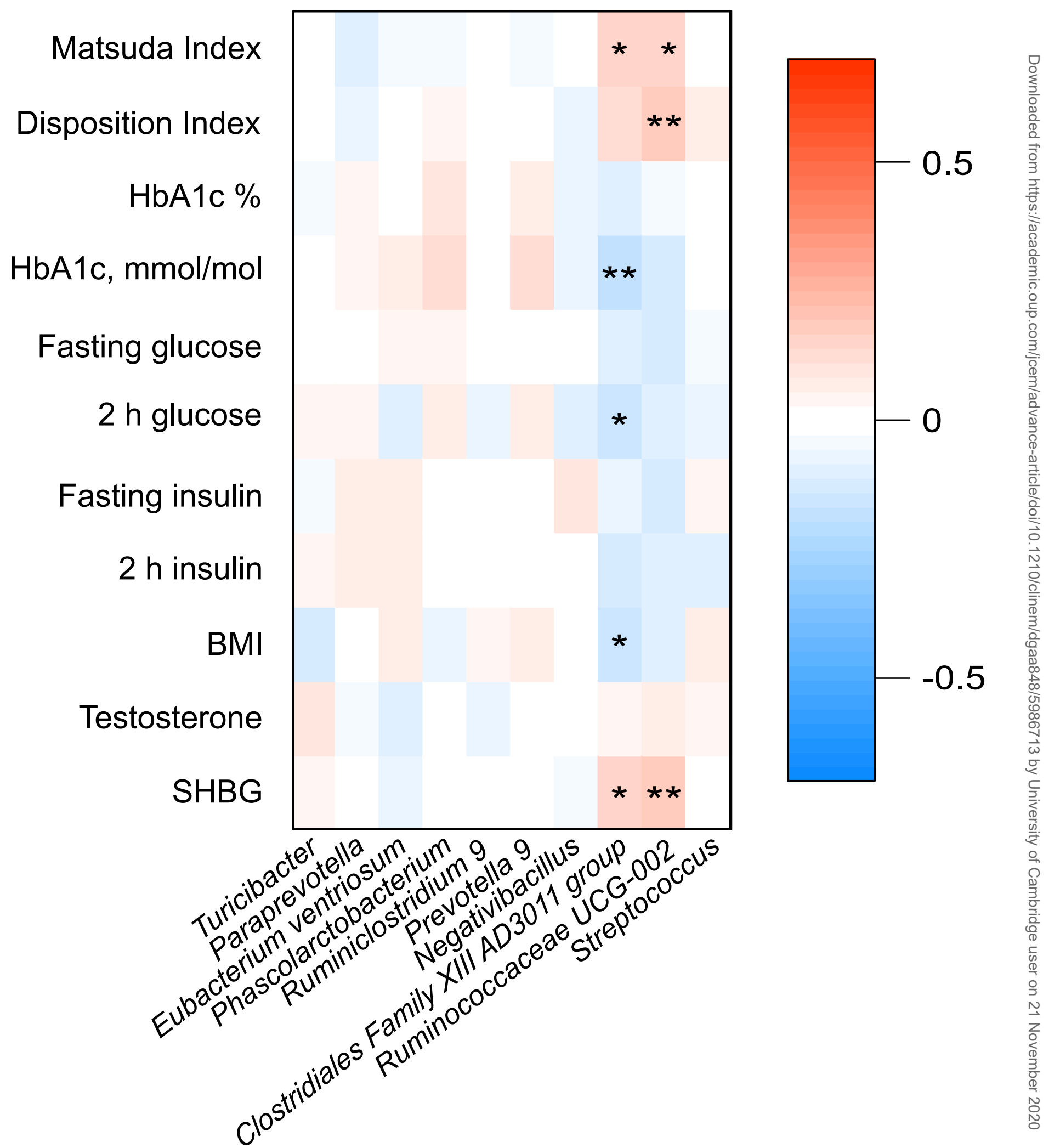



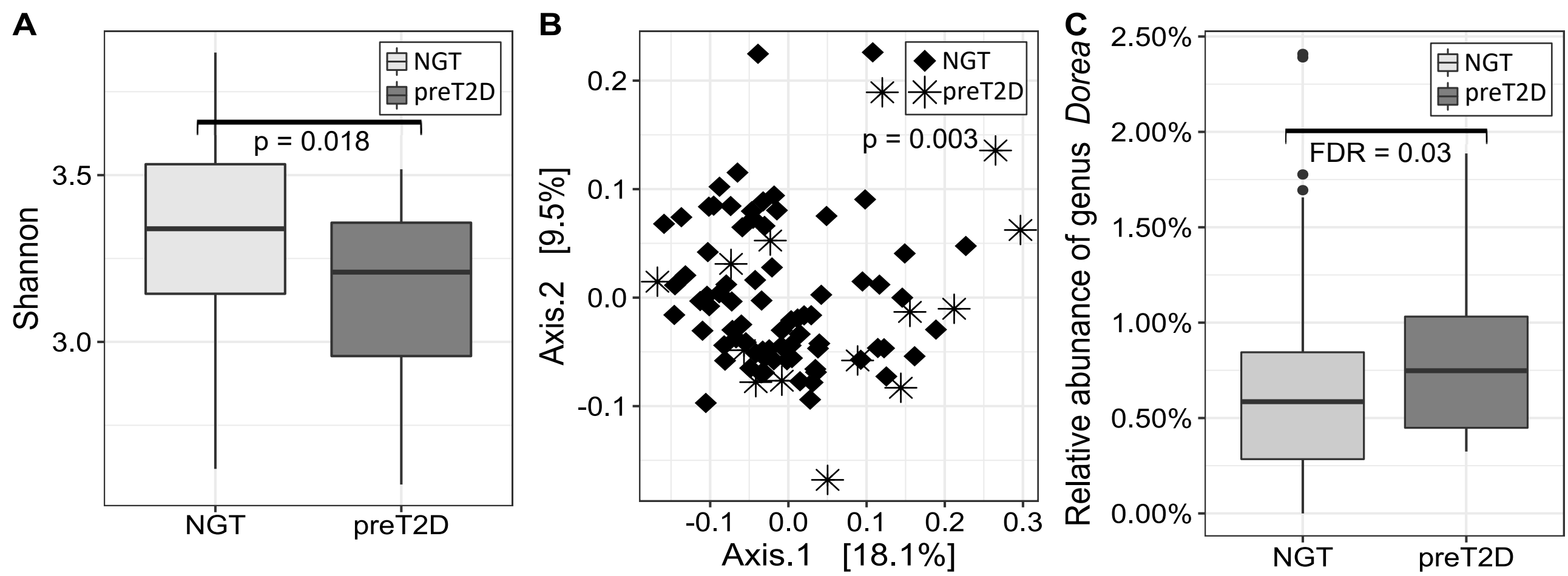